\title{
The perceptual interaction of graphical attributes: Configurality, stimulus homogeneity, and object integration
}

\author{
C. MELODY CARSWELL \\ University of Louisville, Louisville, Kentucky \\ and \\ CHRISTOPHER D. WICKENS \\ University of Illinois, Urbana-Champaign, Illinois
}

\begin{abstract}
Researchers have proposed that graphical efficacy may be determined, in part, by the nature of the perceptual interactions that exist between attributes used to create graphical displays. One extreme type of interaction is integrality, in which two or more physical dimensions are represented as a single psychological dimension in the observer. An alternative type of interaction is configurality, in which a global emergent dimension is available to the observer in addition to the component attributes. Thirteen stimulus sets, each composed of attributes commonly used in the design of graphs, were submitted to the performance-based diagnostics of integrality and configurality. Analyses suggest a continuum of configurality among the present stimulus sets, with little evidence for integral graphical attributes. The configural pattern of results was more common when two identical dimensions were paired (homogeneous stimuli) than when two different dimensions were paired (heterogeneous stimuli). However, there was no evidence that pairs of dimensions belonging to a single object (object integration) were any more configural than dimensions belonging to different objects. Object integration was, however, consistently related to inefficient performance in tasks requiring the filtering of one of two component dimensions.
\end{abstract}

How may geometric properties and color be most effectively employed to communicate quantitative information? What, in short, makes a good graphical display? These questions represent the research agenda for what DeSanctis (1984) calls comparative graphics. One approach to comparative graphics has been to systematically study the accuracy with which we can identify and discriminate stimulus levels along continuous physical dimensions, and this information is routinely presented in introductory discussions of display design (e.g., Hutchingson, 1981; McCormick \& Sanders, 1982). However, this tradition, which emphasizes the coding of individual quantitative variables as individual physical dimensions, has recently been supplemented by interest in the perceptual interactions that may occur when combinations of such dimensions are used to represent multiple variables. In the present paper, we consider the utility of two notions of attribute interaction-dimensional integrality and dimensional configurality - for the prediction of graphical efficacy.

For their programming and technical assistance, we thank Kellie Keifer and Roger Marsh. We also thank Art Kramer, Lester Krueger, and three anonymous reviewers for their comments on earlier versions of this manuscript. Correspondence may be addressed to $\mathrm{C}$. Melody Carswell, Department of Psychology, University of Louisville, Louisville, KY 40292.

\section{Integral and Configural Dimensions}

At an intuitive level, the concepts in integrality and configurality both refer to the subjective unity of a collection of physically specified dimensions. Technically, however, these concepts refer to distinct types of perceptual interactions that may each lead to the impression of unitary wholes. For example, the position of a single point on a graph seems relatively unitary, even though we know that it can also be described more analytically in terms of its horizontal and vertical position. These two attributes, in fact, are frequently used in the design of graphs to represent values of two distinct variables (e.g., a point in a scattergram). In asking whether the two physically defined dimensions of horizontal and vertical position are integral, we would like to know if two-dimensional position is coded and processed directly as a "fundamental unit" of perception. Alternatively, do these two dimensions correspond to distinct perceptual codes? Two physical attributes that correspond to a single perceptual code are integral, whereas two physical attributes that are each associated with distinct perceptual codes are separable (Garner, 1970, 1974).

Configural dimensions differ from integral dimensions in that they maintain separate codes perceptually. However, in contrast to totally separable dimensions, new relational or "emergent" properties are also coded (Garner, 1974, 1978; Pomerantz, 1981; Pomerantz \& 
Garner, 1973; Pomerantz, Sager, \& Stoever, 1977). For example, the orientations of two line segments in a line graph may be considered configural if a relational property, such as the angle formed by the two segments, is also directly available to the observer. Such properties as figural symmetry, closure, and similarity may all be examples of emergent features.

In order to diagnose dimensional interactions at early information-processing stages, researchers have often measured performance in speeded-classification tasks. Garner and co-workers (Garner, 1970, 1974, 1976, 1981; Garner \& Felfoldy, 1970; Garner \& Pomerantz, 1973) have been particularly influential in delineating the performance outcomes associated with integral, separable, and configural dimensions. In the typical procedure, subjects classify stimuli like those in Figure 1 . In this particular stimulus set, two physical dimensions are variedthe vertical positions of the left and right dots. Figure 1 also provides a list of nine tasks a subject might perform. Task 1, for example, would require subjects to give one response to examples of Stimulus $\mathrm{A}$ and an alternate response to examples of Stimulus C. Task 5 would require subjects to make the same response whenever they saw either Stimulus A or Stimulus B, but to make a different response if they saw either Stimulus C or Stimulus D.

Dimensional integrality is diagnosed if classification performance is characterized by filtering interference and redundancy gains (Garner, 1974, 1976; Garner \& Felfoldy, 1970). Filtering interference is demonstrated by comparing performance in baseline discriminability tasks,

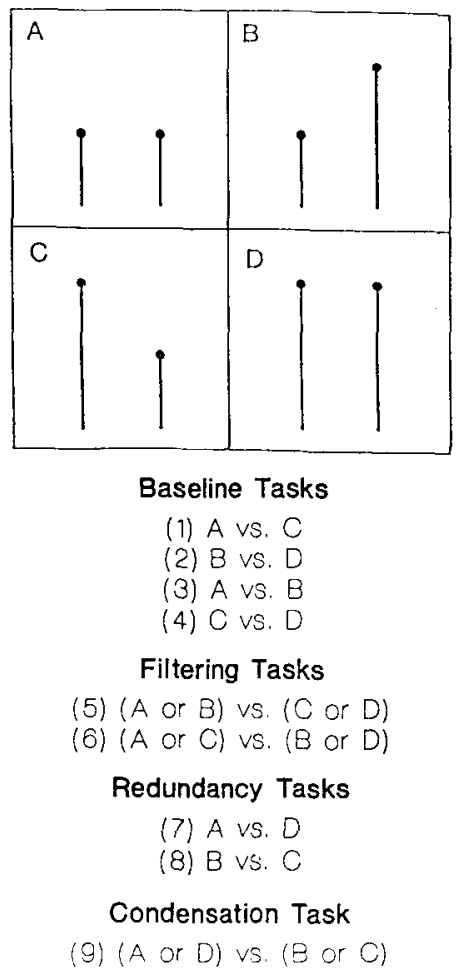

Figure 1. Nine speeded-classification tasks typically used to diagnose integrality, separability, and configurality. such as Tasks 1-4 in Figure 1, to performance in filtering tasks, such as Tasks 5 and 6 . In all of these tasks, classifications can be made by determining the level of only one of the two stimulus dimensions. However, in the baseline tasks, the second dimension remains at a constant value. In the filtering tasks, the second dimension can take on either of its possible values. If this variation of the nontarget dimension disrupts performance relative to the baseline tasks, then filtering interference has occurred. Redundancy gain, on the other hand, occurs when performance in the redundancy tasks (Tasks 7 and 8) is more efficient than performance in the baseline tasks. In the redundancy tasks, the classification can once again be made on the basis of one dimension, but in this case the second dimension varies in association with the first. Garner reasoned that for integral dimensions, the level of both dimensions would necessarily influence performance, even when the task could be performed on the basis of one dimension. For separable dimensions, performance in the filtering and redundancy tasks should be no different from baseline unidimensional performance; the two dimensions do not interact to influence performance.

The diagnosis of configural dimensions, as with integral dimensions, depends on evidence of filtering interference; however, unlike integral dimensions, consistent redundancy gains are not associated with configurality (Pomerantz, 1981; Pomerantz \& Garner, 1973; Pomerantz, Pristach, \& Carson, 1989). In contrast to both integral and separable dimensions, condensation efficiency tends to be high (Pomerantz \& Schwaitzberg, 1975). Task 9 in Figure 1 is an example of a condensation task. Unlike the other eight tasks, the condensation task cannot be performed by using only one of the two dimensions. Thus, for many pairs of dimensions, the condensation task takes longer to complete than any of the other tasks. However, if the dimensions configure, subjects may be able to use an emergent feature as the sole basis for the classification, and thus the decision can be made more quickly than if each parent dimension were being processed sequentially.

\section{Integrality, Configurality, and Comparative Graphics}

Students of comparative graphics have attempted to use the integral/separable classification to predict graphical efficacy in tasks other than simple classification. Such research has been based on the premise that the mandatory perceptual integration of graphical attributes might serve as a processing shortcut, circumventing the resource-intensive logical integration of the information presented by the graph. Jacob, Egeth, and Bevan (1976) used "integral displays" (i.e., graphs composed of integral dimensions) to support performance in identification tasks. Goldsmith and Schvaneveldt (1984) and Goettl, Kramer, and Wickens (1986) compared integral and separable graphs for multicue judgments. Studies of failure detection and diagnosis in process-control scenarios have also been performed using both integral and separ- 
able displays (Carswell \& Wickens, 1987; Casey \& Wickens, 1986; Jones \& Wickens 1986; Petersen, Banks, \& Gertman, 1981). As a general rule, integral displays are related to superior performance only when subjects must process each of the displayed variables in order to make a single response. Separable displays seem to better support tasks requiring information filtering, localization, or performance of several independent tasks simultaneously (see Carswell \& Wickens, 1988, for a review).

However, the labeling of graphs as integral or separable for purposes of graphics research has been largely based on intuitive definitions. In none of the studies have the standard diagnostics of speeded classification been used to classify or rank graphs in terms of their integrality. Accordingly, some authors have questioned whether any of their graphical elements were truly integral (Carswell \& Wickens, 1987; Jacob et al., 1976). Disturbing questions have also come from studies in which integral display benefits would be most strongly predicted, those studies requiring the processing of correlated variables (Casey \& Wickens, 1986; Jones \& Wickens, 1986). The data indicate that integral graphs fail to produce better performance, and may even produce degraded performance, under conditions of high intervariable correlation. This is surprising given that one diagnostic of integral dimensions is redundancy gain in classification tasks. Thus, the relevance of dimensional integrality to comparative graphics has been questioned, and the very existence of integral graphs has been challenged.

As an alternative to using the integral/separable distinction, some researchers have proposed that the presence of emergent features may be critical for determining which graphs provide the user with perceptual shortcuts to logical information integration (e.g., Barnett \& Wickens, 1988; Coury \& Purcell, 1988; Jacob et al., 1976; Sanderson, Flach, Buttigieg, \& Casey, 1989). This emergent features approach to comparative graphics suggests that many of the "integral" displays used in previous studies are actually composed of configural dimensions. One aim of the present study is to evaluate this claim by determining the incidence of integrality and configurality in a sample of stimulus sets representative of the dimensional pairings used in a variety of graphical displays.

A second goal of the present research deals specifically with the concept of configurality and its performancebased diagnostics. If configurality is to gain major explanatory and predictive power within the field of comparative graphics, our methods of detecting configural dimensions must be well established. However, there has been recent concern over the frequent lack of convergence among the more standard diagnostics for integrality (e.g., Cheng \& Pachella, 1984; Pachella, Somers, \& Hardzinski, 1981), which raises similar concerns regarding the more tentative diagnostics for configurality. Initially, we need to be certain that, as proposed by Pomerantz and colleagues (e.g., Pomerantz, 1981; Pomerantz \& Schwaitzberg, 1975), dimensional pairs that are likely to contain global emergent features consistently show filter- ing interference and condensation efficiency, but no redundancy gains.

In the present experiment, we assess the convergence of these proposed diagnostics, as well as the convergence of two additional measures of configurality. Pomerantz and Pristach (1989) have suggested that emergent features may be associated with differential performance in the two redundancy tasks normally included in speededclassification batteries. They argue that such performance differences would result if one classification task could be performed on the basis of a salient emergent feature, but the other task could not. Referring to Figure 1, this measure could be obtained by comparing performance in Tasks 7 and 8. In addition, between-subject variability in filtering interference could be used to detect configurality. This diagnostic is implied in Garner's (1974) definition of configural dimensions as "optionally separable," meaning that subjects may respond either to the global emergent features of the stimulus or to the experimenterdefined parent dimensions. Subjects attending to the more global emergent features would be expected to show greater filtering interference than would subjects who attended to the parent dimensions, resulting in betweensubject variability in filtering performance.

Finally, we address an issue of importance both to comparative graphics and to research on perceptual organization more generally-the identification of stimulus manipulations associated with configural and integral performance patterns. One such manipulation may be the repeated use of the same dimension to create homogeneous stimuli. Figure 1 shows an example of a homogeneous stimulus set since both its dimensions involve positional variation. Heterogeneous stimuli, on the other hand, are formed by pairing dissimilar dimensions. The influence of such homogeneity on dimensional interaction has been explored by Garner (1978), who provided some evidence that homogeneous stimuli tend to be associated with the configural pattern of performance.

A second stimulus manipulation that has received extensive study in comparative graphics is the physical integration of dimensions into a single perceptual object (see Wickens, 1986, for a review). Such object integration results in stimulus sets that are sometimes called "object displays," and are contrasted with multiobject displays that use dimensions of separate objects to communicate multiple quantitative values. Garner (1976) has argued that while attributes of two distinct objects are almost surely separable, attributes of a single object are frequently (but not inevitably) integral or configural. Furthermore, Pomerantz and Schwaitzberg (1975) have demonstrated that configurality effects are stronger with increased spatial proximity between parent dimensions. Since parts of an object are usually located proximally, there may be an increased chance of configurality effects among object displays. Object displays, thus, may benefit (or suffer) from the holistic processing resulting from either integrality or configurality to a greater extent than the traditional multiobject formats. 


\section{The Present Experiment}

The present experiment has three primary goals. First, we address concerns regarding the incidence of integral dimensions in commonly used graphical formats by studying speeded-classification performance in 13 stimulus sets. These stimulus sets were selected to include common graphical attributes, such as linear extent, orientation, and color, and they are combined so as to provide variation in display homogeneity and object integration. Analysis of the speeded-classification results will be used to categorize and thus ascertain the prevalence of integrality, configurality, and separability. A second goal will be to assess the pattern of covariation of the performance outcomes reputedly associated with configurality. Using a principal components analysis, we will determine whether condensation efficiency, filtering interference, redundancy asymmetry, and between-subject filtering variation converge in the predicted manner. Finally, the relation of stimulus homogeneity and object integration to dimensional integrality and configurality will be explored.

\section{METHOD}

\section{Subjects}

Thirteen groups of 9 subjects each performed the battery of speeded-classification tasks. Each group included both men and women, all with normal or corrected-to-normal vision. Any subject' reporting color blindness was screened from the study. The subjects were University of Illinois undergraduates who received class credit for their participation.

\section{Stimuli (Graphical Displays)}

Figure 2 illustrates the 13 stimulus sets used in the present study. Each set includes four stimuli formed by combining two levels of two physical dimensions. The difference between the top and bottom pair of stimuli in each set is the difference between Level 1 and Level 2, respectively, on Dimension 1. Likewise, the difference between the left and right pair of stimuli in each set is the difference between Levels 1 and 2 on Dimension 2 .

The stimulus sets were chosen to be generalizable both to research in comparative graphics, as well as to basic research on dimensional interactions. Two features of the present stimuli increase their relevance to graphical design. First, the physical dimensions or "building blocks" for all stimulus sets were chosen from the set of dimensions described by Cleveland (1985) as basic graphical elements. The dimensions chosen were linear extent, orientation, and color. Second, the method of combining the individual elements was guided by graphical formats in present use in statistics and industry. An introduction to relevant statistical formats is available in Chambers, Cleveland, Kleiner, and Tukey (1983), Tufte (1983), and Wainer and Thissen (1981). Examples of industrial graphics are also available (e.g., Cole, 1986, and Siegel, Goldwyn, \& Friedman, 1971, for medical graphics; Stokes \& Wickens, 1988, for transportation graphics; Woods, Wise, \& Hanes, 1981, for processcontrol graphics).

The stimulus sets were also chosen to increase the likely variety of dimensional interactions obtained. Therefore, Graphs 1-8 in Figure 2 were composed of homogeneous pairings of dimensions, and Graphs 9-12 were composed of heterogeneous pairings. Object integration was also varied in the present sample, with singleobject displays forming a subset of both the homogeneous stimulus sets (Graphs 1, 3, 4, 5, and 6) and the heterogeneous stimulus sets (Graphs 9, 11, and 12). The classification of stimulus sets as consisting of either one or two objects was based on sorting data obtained from 20 subjects in an additional study (Carswell, 1988).
Homogeneous Stimulus Sets

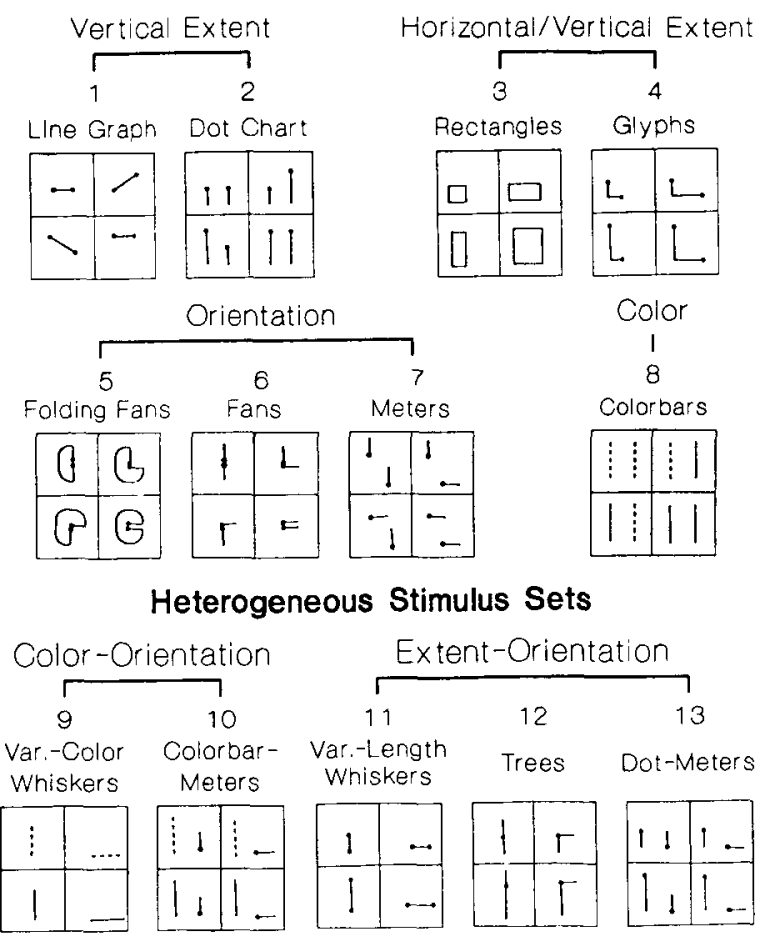

Figure 2. Sample of 13 stimulus sets, each of which was used by an independent group of subjects to perform the nine-task speededclassification battery. For Graphs 8,9 , and 10 , solid lines indicate dark red bars and broken lines indicate brown bars.

Two levels of three dimensions-linear extent, orientation, and color-were the basic building blocks of all 13 stimulus sets. Linear extent was varied for one or both dimensions in Graphs 1, 2, 3, $4,11,12$, and 13. Level 1 was an extent of $1.4 \mathrm{~cm}$, and Level 2 was an extent of $2.6 \mathrm{~cm}$. Orientation was used in eight of the stimulus sets (Graphs 5, 6, 7, 9, 10,11, 12, and 13). Level 1 for this dimension was a vertical line, and Level 2 was a horizontal line. Finally, color was used in three of the stimulus sets (Graphs 8, 9, and 10). The colors used in these displays were amber (Level 1) and red (Level 2), known as "brown" and "dark red" on the IBM graphics adapter color set. These two colors were described by two raters, each reporting normal color vision, as 4.0 Y 6.5/6 (brown) and 7.5 R 4.5/8 (dark red) using the Munsell color system (Munsell Book of Color, 1976).

All displays were centered in the middle of a CRT and were surrounded by a brown box. The box was $5.2 \times 5.2 \mathrm{~cm}$, and subtended a visual angle of approximately $4.29^{\circ}$.

\section{Apparatus}

Stimulus presentation and data collection were controlled by an IBM PC with a Princeton Graphics Master graphics board. The monitor and a chair with arm-mounted keypads were positioned inside a darkened booth.

Tasks

Each subject performed nine different speeded-classification tasks with one of the stimulus sets. Figure 1 illustrates the nine tasks using Graph 2 (the dot charts) as an example. Two unidimensional control tasks, Tasks 1 and 2, were used as baseline measures for all classifications that could be performed on the basis of Dimension 1. Likewise, Tasks 3 and 4 were used as baseline measures for classification of Dimension 2. Tasks 5 and 6 tested the subjects' 
abilities to make classifications based on Dimensions 1 and 2, respectively, when the additional dimension varied randomly from trial to trial. Tasks 7 and 8, on the other hand, required classification of stimuli defined by redundantly paired dimensions. Specifically, Task 7 was a "positive correlation" 'task in which a stimulus composed of the first level of both dimensions was classified against a stimulus composed of the second level of both dimensions. Task 8 was a "negative correlation task" in which a stimulus composed of the first level on Dimension 1 and the second level on Dimension 2 was classified against a stimulus composed of the reverse association. Finally, Task 9 required the subjects to classify stimuli on the basis of the joint values of both dimensions.

\section{Procedure}

The subjects participated in individually administered 2-h sessions. The sessions were composed of four replications of nine blocks of trials. Each block of trials was associated with one of the nine experimental tasks illustrated in Figure 1. The order of blocks was randomized for each subject and replication. Data were collected from only the last three replications.

A single block of $\mathbf{4 0}$ trials was conducted as follows. The experimenter first told the subject which task to perform. As a memory aid, the subjects were given cards with the four stimuli labeled A, B, C, or D. Thus, the experimenter could instruct the subject to, for example, "press the left key if you see either A or D, and press the right key if you see either B or C." A subject receiving these instructions would be prepared to perform Task 9, the condensation task. When the subject was ready, the experimenter initiated a block of trials. A stimulus would appear on the monitor for a maximum of $3 \mathrm{sec}$. If the subject did not respond in that period of time, a "respond faster" message appeared. If the subject made an incorrect response within $3 \mathrm{sec}$, an "error" message appeared. Otherwise, no message appeared and the next trial was initiated $1 \mathrm{sec}$ after the response. After no-response or error trials, the new stimulus was presented $1 \mathrm{sec}$ after the 2-sec message was terminated. After performing 40 trials, the subject was given feedback, including total percent correct and mean correct response times (RTs).The next block of the replication would proceed in a similar manner.

\section{Data Analysis}

Mean RTs for correct responses and percent errors (PEs) were analyzed separately for each of the stimulus sets. These data were submitted to two-factor (nine tasks $\times$ three replications) repeatedmeasure analyses of variance (ANOVAs). Prior to analysis, data points more than three interquartile ranges above the 75th percentile or below the 25 th percentile were eliminated.

The main effect of task was reliable $(p<.01)$ for all analyses, and there was typically a decrease in RTs and PEs across replications $(p<.05)$. Task $\times$ replication interactions were rarely encountered, and when they were obtained, they did not involve the specific comparisons reported below. Thus, only the results of a series of planned comparisons between tasks, jointly used to infer integrality, configurality, or separability, are reported. These comparisons include: (1) mean performance in Tasks 1 and 2 versus performance in Task 5 (filtering interference 1), (2) mean performance in Tasks 3 and 4 versus performance in Task 6 (filtering interference 2), (3) best performance obtained on a control dimension (i.e., either the means of Tasks 1 and 2 or of Tasks 3 and 4) versus performance in Task 7 (redundancy gain 1), (4) best performance obtained on a control dimension (i.e., either the means of Tasks 1 and 2 or of Tasks 3 and 4) versus performance in Task 8 (redundancy gain 2), and (5) mean performance in Tasks 5 and 6 versus performance in Task 9 (condensation efficiency).

The first and second comparisons assess the filtering interference incurred when classification was based on Dimensions 1 and 2, respectively. Comparisons 3 and 4 evaluate the effect of the redun- dant variation of the two dimensions. Both comparisons use as a baseline the better mean performance of the two component dimensions for each subject, following the procedure used by Biederman and Checkosky (1970) for estimating redundancy effects. Finally, Comparison 5 assesses condensation efficiency by comparing condensation performance with mean filtering performance (Tasks 5 and 6).

Diagnosis of integral dimensions depends on evidence of both filtering interference and redundancy gains, whereas diagnosis of separable dimensions relies, predominantly, on evidence of poor condensation efficiency with no filtering interference or redundancy gain. The diagnosis of configurality relies on good condensation performance, with filtering interference but no redundancy gain (and perhaps asymmetric redundancy performance). Because diagnosis of these types of dimensional interactions requires joint inferences based on several comparisons, only individual comparisons significant at the $p<.01$ level are reported.

In addition to subjecting the data to the traditional univariate analysis of dimensional interaction, a multivariate analysis was performed on summary measures from each of the 13 stimulus sets. These summary measures are outlined in Table 1, and include both total and relative discriminability of the two dimensions, redundancy gain, filtering interference, redundancy asymmetry, condensation efficiency, and variability of filtering interference. In order to study the covariance structure of these variables across the 13 stimulus sets, a principal components analysis was performed.

\section{RESULTS AND DISCUSSION}

\section{Traditional Diagnostics}

Filtering performance. Table 2 presents the means of the values used to assess the effects of filtering on classifications of Dimensions 1 and 2. All statistically reliable effects were in the direction of performance decrements, with responses taking longer and being more error-prone when the subjects were required to filter variation in the irrelevant dimension. Reliable increases in RT were obtained for one or both dimensions in seven of the eight object-integrated stimulus sets. These stimulus sets included the line graphs (Graph 1), rectangles (Graph 3), folding fans (Graph 5), fans (Graph 6), variable-color whiskers (Graph 9), variable-length whiskers (Graph 11), and trees (Graph 12). Similar increases in PEs were found for each of these graphs, but only some of these trends were statistically reliable (see Table 2 ). No reliable per-

Table 1

Summary of Variables Used in the Principal Components Analysis of Task Performance Across Graphs

\begin{tabular}{ll}
\hline \multicolumn{1}{c}{ Variable Name } & \multicolumn{1}{c}{ Description } \\
\hline Total Discriminability & $\mathrm{D} 1+\mathrm{D} 2$ \\
Relative Discriminability & $|\mathrm{D} 1-\mathrm{D} 2|$ \\
Redundancy Gain & (Df/R1 + Df/R2)/2 \\
Redundancy Asymmetry & $|(\mathrm{Df} / \mathrm{R} 1-\mathrm{Df} / \mathrm{R} 2)|$ \\
Filtering Interference & (F1/D2 + F2/D2)/2 \\
Filtering Variability & $\mathrm{SD}$ (Filtering Decrement) \\
Condensation Efficiency & $\mathrm{Ds} / \mathrm{C}$ \\
\hline
\end{tabular}

Note-D1 = discriminability (baseline) RT of Dimension 1, D2 = discriminability (baseline) RT of Dimension 2, Df $=$ faster of D1 and D2, $\mathbf{R} 1=\mathrm{RT}$ of first redundancy condition, $\mathbf{R} 2=\mathrm{RT}$ of second redundancy condition, $F 1=R T$ of first filtering condition, $F 2=R T$ of second filtering condition, $\mathrm{C}=\mathrm{RT}$ for condensation task, Ds = slower of D1 and D2. 
Table 2

Analysis of Filtering Interference: Mean Response Time (RT, in msec) and Percent Error (PE) for Baseline and Filtering Classifications with Dimensions 1 and 2 of Each of the 13 Stimulus Sets

\begin{tabular}{|c|c|c|c|c|c|c|c|c|}
\hline \multirow[b]{4}{*}{ Stimulus Set } & \multicolumn{8}{|c|}{ Task } \\
\hline & \multicolumn{4}{|c|}{ Dimension 1} & \multicolumn{4}{|c|}{ Dimension 2} \\
\hline & \multicolumn{2}{|c|}{ Baseline } & \multicolumn{2}{|c|}{ Filtering } & \multicolumn{2}{|c|}{ Baseline } & \multicolumn{2}{|c|}{ Filtering } \\
\hline & RT & $\mathrm{PE}$ & $\overline{R T}$ & $\mathrm{PE}$ & RT & PE & RT & PE \\
\hline \multicolumn{9}{|c|}{ Homogeneous Stimulus Sets } \\
\hline 1-Line Graphs & 311 & 4.91 & $405 \dagger$ & 7.87 & 310 & 5.84 & $404 \ddagger$ & 9.07 \\
\hline 2-Dot Charts & 357 & 3.52 & 373 & 4.45 & 352 & 3.56 & 363 & 3.62 \\
\hline 3-Rectangles & 328 & 3.48 & $372 \dagger$ & 5.46 & 343 & 4.72 & $388 \dagger$ & 6.20 \\
\hline 4-Glyphs & 344 & 3.38 & 348 & 3.66 & 353 & 3.72 & 365 & 4.23 \\
\hline 5-Folding Fans & 349 & 4.58 & $412 \dagger$ & $8.07 *$ & 344 & 5.09 & $405 *$ & 7.82 \\
\hline 6-Fans & 349 & 5.60 & $402 *$ & $8.47 *$ & 343 & 4.24 & $370 *$ & 5.65 \\
\hline 7-Meters & 338 & 6.67 & 339 & 5.83 & 339 & 8.48 & 348 & 6.66 \\
\hline 8-Colorbars & 355 & 5.00 & 399 & 5.37 & 352 & 4.63 & 375 & 5.19 \\
\hline \multicolumn{9}{|c|}{ Heterogeneous Stimulus Sets } \\
\hline 9-V.-C. Whiskers & 338 & 4.40 & $358 *$ & 6.30 & 302 & 2.04 & 302 & 2.22 \\
\hline 10-Colorbar-Meters & 340 & 6.58 & 341 & 6.76 & 333 & 7.09 & 339 & 6.67 \\
\hline 11-V.-L. Whiskers & 333 & 5.65 & $376 \dagger$ & $8.70 \ddagger$ & 306 & 4.31 & $324^{*}$ & 4.90 \\
\hline 12-Trees & 330 & 3.33 & $408 \dagger$ & $7.34 \dagger$ & 325 & 3.35 & $361 \ddagger$ & 4.86 \\
\hline 13-Dot-Meters & 338 & 5.14 & 343 & 5.00 & 342 & 6.39 & 354 & 5.83 \\
\hline
\end{tabular}

Note-Dimension 1 is the leftmost or topmost element in the display, except in Graphs 9, 11, and 12. In these graphs, the first dimension is color (Graph 9) and position (Graphs 11 and 12). V.-C. = variable-color, V.-L. = variable-length. $\quad{ }^{*} p<.01 . \quad \dagger p<.001 . \quad \ddagger p<.0001$.

formance decrements were observed for either dependent variable for any of the stimulus sets composed of two objects.

Redundancy effects. Although reliable filtering interference indicates that some type of dimensional interaction is occurring, such interference alone does not distinguish configural from integral interactions. In order to determine whether any of the stimulus sets are composed of integral dimensions, performance facilitation in a redundancy task must also be demonstrated. Table 3 presents the results of the two comparisons that assessed redundancy effects-the comparison of each subject's faster control performance with performance in each of the two redundancy tasks.

Redundancy effects were obtained with three of the stimulus sets that showed filtering interference. However, contrary to the pattern diagnostic of integral interaction, none of these effects were facilitative. The line graphs (Graph 2) were associated with increased RTs and PEs in the redundancy condition requiring classifications of positively correlated dimensions. The trees (Graph 12) also showed reliably increased RTs in this condition, but the PEs showed only a nonreliable upward trend. The folding fans, in contrast, were associated with increased RTs and PEs in the redundancy condition requiring classification of negatively correlated dimensions. In addition to these stimulus sets, two others were associated with performance decrements in one or the other redundancy task. The glyphs (Graph 4) revealed increased PEs in the positive correlation condition, and the dot-meters (Graph 13) revealed increased RTs in the negative correlation condition.
In summary, only detrimental redundancy effects were obtained. Redundancy-related performance decrements would not be anticipated with either integral or separable dimensions; however, Pomerantz and Pristach (1989) have suggested that configural dimensions might produce such a decrement. They reasoned that a decrement might result when covariation of the dimensions makes it impossible to use an emergent feature that could be used in

Table 3

Analysis of Redundancy Gains: Mean Response Time (RT, in msec) and Percent Error (PE) for Faster Baseline, Positive Redundancy, and Negative Redundancy Classifications with Each of the 13 Stimulus Sets

\begin{tabular}{|c|c|c|c|c|c|c|}
\hline \multirow[b]{3}{*}{ Stimulus Set } & \multicolumn{6}{|c|}{ Task } \\
\hline & \multicolumn{2}{|c|}{$\begin{array}{c}\text { Faster } \\
\text { Baseline }\end{array}$} & \multicolumn{2}{|c|}{$\begin{array}{c}\text { Positive } \\
\text { Redundancy }\end{array}$} & \multicolumn{2}{|c|}{$\begin{array}{c}\text { Negative } \\
\text { Redundancy }\end{array}$} \\
\hline & RT & PE & RT & $\mathrm{PE}$ & RT & $\mathrm{PE}$ \\
\hline \multicolumn{7}{|c|}{ Homogeneous Stimulus Sets } \\
\hline 1-Line Graphs & 305 & 4.72 & $335^{*}$ & $8.15^{*}$ & 301 & 4.08 \\
\hline 2-Dot Charts & 349 & 2.92 & 348 & 3.70 & 335 & 2.69 \\
\hline 3-Rectangles & 326 & 3.11 & 332 & 4.73 & 325 & 4.54 \\
\hline 4-Glyphs & 338 & 2.04 & 337 & $3.15^{*}$ & 346 & 3.29 \\
\hline 5-Folding Fans & 343 & 4.13 & 332 & 3.80 & $359 *$ & 6.5 \\
\hline 6-Fans & 343 & 3.96 & 329 & 4.91 & 335 & 6.02 \\
\hline 7-Meters & 335 & 6.06 & 332 & 6.94 & 339 & 6.39 \\
\hline 8-Colorbars & 350 & 4.17 & 333 & 5.28 & 338 & 4.07 \\
\hline \multicolumn{7}{|c|}{ Heterogeneous Stimulus Sets } \\
\hline 9-V.-C. Whiskers & 301 & 1.53 & 297 & 2.29 & 298 & 1.48 \\
\hline 10-Colorbar-Meters & 324 & 5.50 & 335 & 5.65 & 332 & 7.04 \\
\hline 11-V.-L. Whiskers & 305 & 4.07 & 307 & 4.35 & 306 & 4.26 \\
\hline 12-Trees & 319 & 2.56 & $337^{*}$ & 3.89 & 306 & 3.03 \\
\hline 13-Dot-Meters & 331 & 4.03 & 336 & 5.19 & $348^{*}$ & 5.65 \\
\hline
\end{tabular}

Note-V.-C. = variable-color, V.-L. = variable-length. $\quad{ }^{*} p<.01$. 
many of the other tasks. They also suggested that such a decrement is likely to occur in only one of the two redundancy conditions, resulting in an asymmetry of redundancy performance. To determine if the present instances of redundancy-related decrements were consistent with this configural pattern, performance in the two redundancy conditions was compared for each of the five stimulus sets showing decrements. For all three of the graphs that had demonstrated both filtering and redundancy-performance decrements, reliable asymmetries in redundancy performance were obtained. The line graphs revealed an asymmetry for both RT $[F(1,8)=10.94, p<.01]$ and PE $[F(1,8)=19.81, p<.002]$, with the negative redundancy condition having lower RTs and PEs. Similarly, the trees (Graph 12) showed reliably superior RT performance for the positive rather than the negative redundancy condition $[F(1,8)=13.20, p<.007]$. The folding fans, on the other hand, showed superior RT performance in the negative redundancy condition $[F(1,8)=20.81, p<$ $.002]$. Redundancy asymmetries were obtained for neither the glyphs (Graph 4) nor the dot-meters (Graph 13).

Condensation performance. Table 4 presents mean filtering and condensation performance. Most of the contrasts between these conditions (10 of 13 for RTs, and 8 of 13 for PEs) revealed reliable performance decrements for condensation. The stimulus sets not associated with decrements were mainly composed of homogeneous pairs of dimensions: the line graphs (Graph 1), the rectangles (Graph 3), the folding fans (Graph 5), and the colorbars (Graph 8). In addition, the heterogeneous trees (Graph 12) failed to show reliably increased PEs, although increased RTs were obtained.

Discussion. The pattern of results obtained for several of the stimulus sets indicates that their component dimensions interact perceptually. In particular, the line graphs

Table 4

Analysis of Condensation Efficiency: Mean Response Time (RT, in msec) and Percent Error (PE) for Filtering and Condensation Classifications with Each of the 13 Stimulus Sets

\begin{tabular}{|c|c|c|c|c|}
\hline \multirow[b]{3}{*}{ Graph } & \multicolumn{4}{|c|}{ Task } \\
\hline & \multicolumn{2}{|c|}{ Mean Filtering } & \multicolumn{2}{|c|}{ Condensation } \\
\hline & RT & $\mathrm{PE}$ & RT & $\mathrm{PE}$ \\
\hline \multicolumn{5}{|c|}{ Homogeneous Stimulus Sets } \\
\hline 1-Line Graphs & 405 & 8.47 & 362 & 9.08 \\
\hline 2-Dot Charts & 368 & 4.03 & $425 \dagger$ & $6.85^{*}$ \\
\hline 3-Rectangles & 380 & 5.83 & $419 \dagger$ & 6.86 \\
\hline 4-Glyphs & 357 & 3.95 & $482 \ddagger$ & $6.86 \ddagger$ \\
\hline 5-Folding Fans & 408 & 7.97 & 417 & 8.52 \\
\hline 6-Fans & 386 & 7.06 & $421^{*}$ & $10.74 *$ \\
\hline 7-Meters & 344 & 6.25 & $462 \ddagger$ & $12.78 \ddagger$ \\
\hline 8-Colorbars & 387 & 5.28 & 400 & $8.89^{\circ}$ \\
\hline \multicolumn{5}{|c|}{ Heterogeneous Stimulus Sets } \\
\hline 9-V.-C. Whiskers & 330 & 4.26 & $524 \ddagger$ & $9.63 *$ \\
\hline 10-Colorbar-Meters & 340 & 6.72 & $625 \dagger$ & $14.07 *$ \\
\hline 11-V.-L. Whiskers & 350 & 6.80 & $563 \ddagger$ & $14.63 \dagger$ \\
\hline 12-Trees & 385 & 6.10 & $524 \ddagger$ & 9.49 \\
\hline 13-Dot-Meters & 349 & 5.42 & $599 \ddagger$ & $13.89 *$ \\
\hline
\end{tabular}

Note-V.-C. = variable-color, V.-L. = variable-length. $\quad{ }^{*} p<$ .01. $\dagger p<.001$. $\neq p<.0001$.
(Graph 1) and the folding fans (Graph 5) both showed the pattern of outcomes associated with configural dimensions. They each showed filtering decrements and failed to show redundancy gains or condensation decrements. The trees (Graph 12) also showed this configural pattern, with the exception that condensation RTs were reliably longer than filtering RTs. In addition, following the suggested configurality diagnostic of Pomerantz and Pristach (1986), all three of these graphs showed asymmetric redundancy performance. In contrast, none of the stimulus sets produced the filtering interference and redundancy facilitation typical of integral dimensions.

Just as there were two stimulus sets that showed the prototypical pattern of configurality, two stimulus sets showed a perfectly separable pattern of results. The meters (Graph 7) and the colorbar-meters (Graph 10) provided no evidence of filtering interference or redundancy gain, and were associated with poor condensation performance relative to filtering. Other stimulus sets were associated with patterns of performance that imperfectly fit the category of separability. For example, the glyphs (Graph 4) and the dot-meters (Graph 13) showed the separable pattern of results except that, for each, one of the two redundancy conditions resulted in a reliable performance interference. The fans (Graph 6), the variablecolor whiskers (Graph 9), and the variable-length whiskers (Graph 11) also showed condensation losses and failed to show redundancy gain; however, all three showed filtering decrements. The colorbars (Graph 8), on the other hand, were not associated with filtering interference or redundancy gains, nor were they associated with poor condensation performance.

The two remaining stimulus sets-the dot charts (Graph 2) and the rectangles (Graph 3)-showed inconsistencies both with the present diagnostic categories and with previous speeded-classification results. The rectangles, for example, showed filtering interference but no redundancy gains. Felfoldy (1974), however, found that sorting of the height and width of rectangles yielded both filtering interference and redundancy facilitation, the performance pattern typical of integrality. It should be noted, however, that in one part of his study he did fail to obtain reliable redundancy gains. Furthermore, Weintraub (1971) provides some evidence that the height and width of rectangles may be configural rather than integral. He found that absolute judgments of rectangles were better when their heights and widths were negatively rather than positively correlated. Only a trend toward such asymmetric redundancy performance was obtained in the present study. However, the rectangles did show the relatively high condensation accuracy that may be associated with the presence of emergent features.

The present results for the dot charts (Graph 2) failed to replicate the data from Lockhead and King (1977), who also used pairs of parallel lines that each varied in height as a stimulus set. Their findings were suggestive of configurality with condensation performance comparable to or better than filtering performance. Our data indicate that 
condensation performance was reliably worse than filtering performance; however, condensation performance was far better than for many of the other graphs in the sample. In addition, our data show a reliable asymmetry between the two redundancy conditions, which provides some evidence for configurality. Our failure to obtain the highly efficient condensation performance reported by Lockhead and King may have resulted from the greater distance between the elements in our stimulus set. The two lines used by Lockhead and King were only $6 \mathrm{~mm}$ apart, subtending approximately $1^{\circ}$ if viewed from a distance of $1 \mathrm{ft}$. The present stimuli subtended approximately $2^{\circ}$. The poorer condensation performance obtained in the present study would be consistent with the results of Pomerantz and Schwaitzberg (1975), who found a dilution of configurality effects with increases of distance between stimulus elements from $1^{\circ}$ to $2^{\circ}$.

A third anomaly in the present data is the association of several stimulus sets with redundancy interference. However, when the line graphs (Graph 1), folding fans (Graph 5), and trees (Graph 12) are considered, additional evidence suggests that these redundancy losses may have resulted from the configural nature of the stimuli, with one of the redundancy conditions disallowing the use of an emergent feature upon which classification might be based in the other tasks. However, with regard to the glyphs (Graph 4) and the dot-meters (Graph 13), there is no additional evidence of configurality, and the finding of redundancy-related losses is somewhat more puzzling. The results of a simulation study by Miller and Lopes (1988) may provide one explanation for these data. These authors argued that while it is necessary to estimate the true faster baseline (unidimensional) RT in order to test some models of redundancy facilitation, the manner in which this faster baseline is generally estimated may lead to systematic biases. In particular, they demonstrated that the commonly used practice of picking the lower mean RT of two experimental conditions may lead to an underestimation of the true lower mean for each subject. An underestimation of the appropriate baseline may, in turn, lead to artifactual redundancy losses.

\section{Principal Components Analysis}

The traditional analysis of speeded-classification results treats the diagnosis of dimensional interaction in a discrete manner: dimensions interact in either one or another specific way. However, as reflected in the above analyses, a number of stimulus sets do not fall perfectly into any one of the present categories of dimensional interaction. One method of handling such outcomes has been to expand the taxonomy of dimensional interactions to include such categories as asymmetric separability (Garner, 1976). Alternatively, some researchers (e.g., Garner, 1974; Smith \& Kilroy, 1979) have suggested that dimensional interaction may be conceptualized as a continuum, with noninteractive or mandatorily separable dimensions forming one endpoint, and strongly interactive dimensions at the other extreme. The present analysis begins with the assumption that dimensional interaction varies continu-
Table 5

Unrotated Factor Pattern for the First Two Principal Components Derived from the Correlation Matrix of the Seven Summary Performance Measures

\begin{tabular}{lcc}
\hline \multicolumn{1}{c}{ Summary Measure } & Component 1 & Component 2 \\
\hline Filtering Variability & .91 & .19 \\
Redundancy Asymmetry & .91 & -.20 \\
Filtering Interference & .89 & -.37 \\
Condensation Efficiency & .68 & .63 \\
Total Discriminability & -.18 & .85 \\
Redundancy Gain & -.13 & .74 \\
Relative Discriminability & -.44 & -.30 \\
\hline
\end{tabular}

Note-Eigenvalues $=3.17$ for Component 1 and 2.03 for Component 2 .

ously from strong to weak levels, and attempts to determine if the attribute interactions obtained in the present set of graphs can be described by a dimension of variation that is a composite of the performance outcomes indicative of configurality or integrality.

Table 5 presents the unrotated factor patterns for the first two principal components. These two components, with eigenvalues of 3.17 and 2.03 , accounted for $74 \%$ of the standardized sample variation. Inspection of the loadings on the first component revealed a cluster of the four variables that are purported to diagnose configurality: condensation efficiency, asymmetrical redundancy performance, filtering interference, and between-subject variability in filtering. Furthermore, redundancy gain received a relatively low loading, which, along with the presence of redundancy asymmetries, is critical to distinguish integrality from configurality. Thus, the first component seems to be a general configurality component.

Baseline unidimensional discriminabilities (i.e., long baseline RTs) loaded most heavily on the second component. In addition, redundancy gain and condensation efficiency were highly weighted. It is unlikely that this component represents integrality, because filtering interference received a negative loading. Rather, it is likely that subjects using graphs that have low unidimensional discriminabilities would attempt to utilize any emergent or relational property of the graph as a whole to overcome this handicap. The relative weighting on condensation performance suggests that this redundancy gain was in fact more common in graphs with salient emergent features.

Scores on the first component are plotted against those on the second component in Figure 3. This plot shows that the line graph (Graph 1) and the folding fans (Graph 5) are strongly configural, whereas the colorbarmeters (Graph 10), the dot-meters (Graph 13), the variable-length whiskers (Graph 11), and the variablecolor whiskers (Graph 9) are all very nonconfigural. Variation along the second component suggests that the colorbars and fans are both associated with slow unidimensional classifications, but both show relatively good redundancy and condensation performance. The lines, trees, and variable-length whiskers, on the other hand, are all associated with relatively fast unidimensional classifications.

Two final analyses were performed to assess the relationship of the derived configurality dimension to stimulus homogeneity and object integration. A point-biserial 


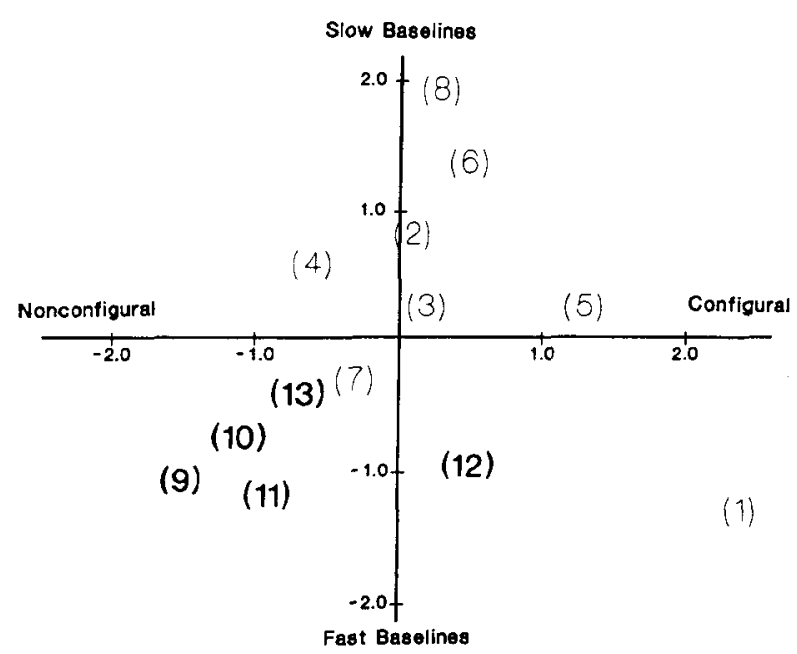

Figure 3. Component scores for the first (horizontal axis) and second (vertical axis) principal components for each of the 13 stimulus sets. Numbers in parentheses correspond to the identification numbers of the stimulus sets. Boldface numbers indicate heterogeneous sets.

correlation between homogeneity and configurality revealed a positive relationship $(r=.52, p=.06)$. Thus, homogeneous displays were generally more likely to be configural, further supporting the findings of Garner (1978). No such relationship was found between object integration and the first principal component, although object displays were more likely than multiobject displays to be associated with filtering interference $\left(\chi^{2}=9.53\right.$, $p<.05)$.

\section{GENERAL DISCUSSION}

\section{Integrality versus Configurality}

An objective of the present paper was to determine which of the alternative conceptualizations of attribute interaction best characterized a sample of graph-like stimulus sets. Do most of these stimulus sets vary along a continuum of configurality, or are they better described as either integral or separable? Using the pattern of performance in nine speeded-classification tasks as a diagnostic, none of the 13 stimulus sets appeared to contain integral dimensions. However, two stimulus sets clearly contained separable dimensions, and two others contained configural dimensions. The nine remaining graphs were associated with performance that failed to fit perfectly into the categories of integrality, configurality, or separability as proposed by Garner, Pomerantz, and colleagues (e.g., Garner, 1974, 1976, 1981; Pomerantz \& Garner, 1973; Pomerantz \& Schwaitzberg, 1975).

Our failure to find a single instance of integrality suggests that the results of many comparative graphics studies may be influenced more directly by other types of dimensional interaction, perhaps by configurality. Although this assertion depends largely on the extent to which the present stimulus sets are representative of previously studied graphs, there are other logical reasons to believe that graphs are unlikely to contain integral dimensions. It seems unlikely that a display designer who was asked to represent some number of variables on a single graph would spontaneously choose dimensions that were not readily analyzable. Thus, the perceptual-generative act of creating a graph may bias the choice of dimensions to those that are at least optionally separable (i.e., configural), if not mandatorily so. The process of choosing display elements should be distinguished from the process of stimulus choice exercised by the scientist wishing to demonstrate dimensional integrality.

Because the choice of the present stimuli was dictated by concerns that they represent common pairings of graphical elements, these data cannot be used as further evidence that "integral dimensions may be a myth" (Cheng \& Pachella, 1984, p. 302). However, the present data may be relevant to the alternative conceptualization of dimensional interaction proposed by these authors. Cheng and Pachella (1984) and Pachella et al. (1981) hold that performance with many supposedly integral dimensions can be explained by looking at the degree of correspondence between physically defined and psychologically separable dimensions. This proposition predicts "degrees of nonseparability," asymmetric redundancy performance, and redundancy loss, all of which were obtained in the present study. However, this conceptualization seems to overlap greatly with the notion of configural dimensions as an intermediate type of mapping between mandatorily integral and separable dimensions. Thus, in essence, Cheng and Pachella argue that many intuitively integral dimensions may be more accurately described as configural, an argument that is consistent within the limits of the present sample of stimuli.

By assuming that graphical displays are likely to utilize configural rather than integral dimensions to represent quantitative variables, several inconsistent findings in the comparative graphics literature become interpretable. Casey and Wickens (1986) and Jones and Wickens (1986) both predicted relatively better performance from an "integral" display when variables were correlated rather than when they were uncorrelated. Instead, these authors found that performance remained stable or even declined with intercorrelation of the variables represented. If it is assumed that these stimulus sets (faces and polygons) varied in their configural dimensions, then no such advantage would have been predicted. As we have witnessed with several of the present stimulus sets, redundancy losses can sometimes occur with configural displays.

\section{The Measurement of Configurality}

If the concept of dimensional configurality is to be of use either to theories of graphical perception specifically or to research on perceptual organization more generally, then we must demonstrate that the various operational definitions of the term do converge. Our analysis of the covariance structure of several scales, including purported measures of configurality and integrality as well as mea- 
sures of unidimensional discriminability, revealed a convergence of the configurality measures. The principal component of the correlation matrix for these measures may be described as a combination of (1) filtering interference, (2) condensation efficiency, (3) redundancy asymmetry, and (4) filtering variability. Redundancy gains and unidimensional discriminabilities, on the other hand, contributed little to this component.

Pomerantz (1981) has suggested that condensation efficiency with filtering decrements and without redundancy gain indicate configurality. More recently, Pomerantz and Pristach (1989) have suggested that configural dimensions will show asymmetries between performance in the tasks that require sorting of redundantly paired attributes. The present data support the association of all these measures. In addition, the data show an association of these measures with the performance outcome implied by Garner's (1974) definition of configurality as optional separability. That is, if configural dimensions allow some degree of strategic processing, between-subject variation in level of filtering interference would be expected. In fact, the stimulus sets that tended to show all the other signs of high configurality also tended to have large intersubject variability in filtering interference. These results are in agreement with the work of Treisman and Paterson (1984), who showed that there was consistency in the ability of some subjects to use an emergent feature over several different tasks. Within a given task, then, we might expect very different experimental results from subjects who make use of such features and from those who do not.

\section{Stimulus Homogeneity}

The present results replicate the findings of Garner (1978), who showed that attribute similarity (or homogeneity) was related to configurality. Garner used as attributes either brackets or parentheses, each of which could be either left-opening or right-opening. Homogeneous pairings (two brackets or two parentheses) showed stronger configurality effects than did heterogeneous pairs (a bracket with a parenthesis). In the present experiment, four of the five heterogeneous stimulus sets were the least configural of all the displays. The only heterogeneous display that seemed to show some configurality was the tree display. This suggests that homogeneity may serve as one rule of thumb for assessing configurality.

However, it should be noted that the homogeneous stimuli varied greatly in degree of configurality. We must look for further stimulus characteristics to account for this variation. A possibility, previously studied by Pomerantz and Schwaitzberg (1975), is spatial proximity. For example, the fans (Graph 6) are more configural than the spatially separate meters (Graph 7). Another possibility is similarity of orientation, previously studied for parenthesis pairs by Pomerantz and Garner (1973). Thus, the parallel dot charts (Graph 2) are more configural than the glyphs (Graph 4), which are set at a $90^{\circ}$ angle to one another.

\section{Object Integration}

Finally, our data may indirectly address the conflicting evidence regarding the superiority of object displays over multiobject formats (e.g., Coury \& Purcell, 1988; Sanderson et al., 1989; Wickens, 1986). The present findings indicate that object displays can be either configural (e.g., line graphs) or nonconfigural (e.g., variable-length whiskers). If the utility of an object display is ultimately dependent on its configurality, then every object display would not be expected to yield performance benefits relative to every multiobject display. Of the present stimulus sets, the rectangles (Graph 3) are the graphs most similar to the object display used by Carswell and Wickens (1987) and Sanderson et al. (1989), as well as to the "configural display" of Coury and Purcell (1988). The dot charts (Graph 2), on the other hand, are representative of the bar charts used in all three experiments. As indicated in Figure 3, neither of these stimulus sets is distinctly more configural than the other. Thus, if configurality is the determining factor in graphical efficacy for the particular tasks studied, then the mixed demonstrations of object-display advantages (e.g., Carswell \& Wickens, 1987) and object-display disadvantages (e.g., Coury \& Purcell, 1988; Sanderson et al., 1989) should not be surprising. It is likely that small differences in the proximity or arrangement of elements in the various studies could vary the configurality and thus the superiority of one graph over the other. In support of this notion, Sanderson et al. (1989) have shown that manipulating the mapping of variables to bars in a bar graph results in varying performance advantages for that graph, presumably due to the differential salience and task-relevance of the emergent feature(s) produced.

Although much remains to be learned about exactly when object displays will provide for enhanced performance, the present data provide some clear-cut evidence for when object displays are likely to be detrimental to performance. For all but one of the object displays in the present sample, the subjects were unable or were strategically disinclined to focus attention on one of two varying graphical elements. For none of the multiobject displays was this a problem. Thus, as suggested by Wickens and Andre (1988) and Carswell and Wickens (1987), object displays should be avoided when the graphical task requires focusing on component dimensions.

\section{CONCLUSIONS}

The present data suggest that measures of attribute configurality may provide a theoretically useful method of categorizing graphical formats. This conclusion is based on a failure to find one instance of true dimensional integrality in a sample of 13 stimulus sets, each representing a simple graphical format. These stimulus sets included single-object and multiobject displays, as well as homogeneous and heterogeneous stimuli. Although the term "integrality" has been used frequently in the litera- 
ture, "configurality" may be more appropriate and may more accurately reflect the processing advantages and disadvantages of a variety of graphs.

In addition, several suggested measures of configurality were found to converge in a predicted fashion. An overall configurality score, created from the weighted sum of these measures, was also shown to be related to stimulus homogeneity. No strong relationship was found between this composite score and object integration. However, the classification of a graph as a single-object display did seem to be related to the difficulty subjects encountered in trying to selectively attend to only one of the graph's attributes. In addition to exploring the relationship of configurality to graphical efficacy in a variety of tasks, further research should attempt to determine what manipulations, in addition to stimulus homogeneity, result in variations in dimensional configurality.

\section{REFERENCES}

BARNeTt, B. J., \& WiCkENS, C. D. (1988). Display proximity in multicue information integration: The benefit of boxes. Human Factors, 30, $15-24$.

Biederman, I., Checkosky, S. F. (1970). Processing redundant information. Joumal of Experimental Psychology, 83, 486-490.

CARSWELl, C. M. (1988). Graphical information processing: The effect of proximity compatibility. Unpublished doctoral dissertation, University of Illinois, Urbana-Champaign.

Carswell, C. M. , \& Wickens, C. D. (1987). Information integration and the object display: An interaction of task demands and display superiority. Ergonomics, 30, 511-528.

Carswell, C. M., Wickens, C. D. (1988). Comparative graphics: History and applications of perceptual integrality theory and the proximity compatability hypothesis (Technical Report ARL-88-2/AHEL88-1). Savoy, IL: Aviation Research Lab, Institute of Aviation, University of Illinois.

CASEY, E. J., \& WICKENS, C. D. (1986). Visual display representation of multidimensional systems (Tech. Rep. CPL-86-2/MDA903-83-K0255). Champaign: Cognitive Psychophysiology Laboratory, University of Illinois.

Chambers, J. M., Cleveland, W. S., Kleiner, B., Tukey, P. A. (1983). Graphical methods for data analysis. Belmont, CA: Wadsworth.

Cheng, P. W., \& PAChella, R. G. (1984). A psychophysical approach to dimensional separability. Cognitive Psychology, 16, 279-304.

Clevveland, W. S. (1985). The elements of graphing data. Monterey, CA: Wadsworth.

Cole, W. G. (1986). Medical cognitive graphics. Proceedings of the Association for Computing Machinery, SIGCHI '86.

Coury, B. G., Purcell, J. (1988). The bargraph as a configural and separable display. Proceedings of the Human Factors Society 32nd annual meeting (pp. 1361-1365). Santa Monica, CA: Human Factors Society.

DESANCTIS, G. (1984). Computer graphics as decision aids: Directions for research. Decision Sciences, 15, 463-487.

Felfoldy, G. L. (1974). Repetition effects in choice reaction time to multidimensional stimuli. Perception \& Psychophysics, 15, 453-459.

GARNER, W. R. (1970). The stimulus in information processing. American Psychologist, 25, 350-358.

GARNER, W. R. (1974). The processing of information and structure. Hillsdale, NJ: Erlbaum.

GARNER, W. R. (1976). Interaction of stimulus dimensions in concept and choice processes. Cognitive Psychology, 8, 98-123.

GARNER, W. R. (1978). Selective attention to attributes and to stimuli. Journal of Experimental Psychology: General, 107, 287-308.

GARNER, W. R. (1981). The analysis of unanalyzed perceptions. In
M. Kubovy \& J. R. Pomerantz (Eds.). Perceptual organization (pp. 119-139). Hillsdale, NJ: Erlbaum.

GARNER, W. R., \& Felfoldy, G. L. (1970). Integrality of stimulus dimensions in various types of information processing. Cognitive Psychology, 1, 225-241.

GoettL, B. P., Kramer, A. F., \& Wickens, C. D. (1986). Display format and the perception of numerical data. Proceedings of the Human Factors Society 30th Annual Meeting (pp. 450-454). Santa Monica. CA: Human Factors Society.

Goldsmith, T., Schvaneveldt, R. (1984). Facilitating multi-cue judgments with integral information displays. In J. Thomas \& M. Schneider (Eds.), Human factors in computer systems (pp. 243270). Norwood, NJ: Ablex.

Hutchingson, R. D. (1981). New horizons for human factors in design. New York: McGraw-Hill.

JACOB, R. J. K., EgETH, H. E., \& BeVAN, W. (1976). The face as a data display. Human Factors, 18, 189-200.

JONES, P., \& WICKENS, C. D. (1986). The display of multivariate information: The effects of auto- and cross-correlation, display format, and reliability (Tech. Rep. CPL-86-5). Champaign: Cognitive Psychophysiology Lab, University of Illinois.

LOCKHEAD, G. R., KING, M. C. (1977). Classifying integral stimuli. Journal of Experimental Psychology: Human Perception \& Performance, $3,436-443$.

MCCoRmick, E. J., SANDERS, M. S. (1982). Human factors in engineering and design. New York: McGraw-Hill.

MilleR, J., \& LOPES, A. (1988). Testing race models by estimating the smaller of two true mean or true median reaction times: An analysis of estimation bias. Perception \& Psychophysics, 44, 513-524.

Munsell book of color (1976). Baltimore: Koll Morgan.

Pachella, R. G., Somers, P., Hardzinski, M. (1981). A psychophysical approach to dimensional integrality. In D. Getty \& J. Howard (Eds.), Visual and auditory pattern recognition (pp. 107-126). Hillsdale, NJ: Erlbaum.

Petersen, R. J., Banks, W. W., * Gertman, D. I. (1981). Performance-based evaluation of graphic displays for nuclear power plant control rooms. Proceedings of the Conference on Human Factors in Computing Systems (pp. 182-189). Gaithersburg, MD: Association for Computing Machinery.

Pomerantz, J. R. (1981). Perceptual organization in information processing. In M. Kubovy \& J. R. Pomerantz (Eds.), Perceptual organization (pp. 141-180). Hillsdale, NJ: Erlbaum.

Pomerantz, J. R., \& GARNer, W. R. (1973). Stimulus configuration in selective attention tasks. Perception \& Psychophysics, 14, 565-569.

Pomerantz, J. R., \& Pristach, E. A. (1989). Emergent features, attention, and perceptual glue in visual form perception. Journal of Experimental Psychology: Human Perception \& Performance, 15 , 635-649.

Pomerantz, J. R., Pristach, E. A., \& Carson, C. E. (1989). Attention and object perception. In B. E. Shepp \& S. Ballesteros (Eds.), Object perception: Structure and process (pp. 53-89). Hillsdale, NJ: Erlbaum.

Pomerantz, J. R., Sager, L. C., \& Stoever, R. J. (1977). Perception of wholes and of their component parts: Some configural superiority effects. Journal of Experimental Psychology: Human Perception \& Performance, 3, 422-435.

Pomerantz, J. R., Schwattzeerg, S. D. (1975). Grouping by proximity: Selective attention measures. Perception \& Psychophysics, 18, 355-361.

Sanderson, P. M., Flach, J. M., Buttigieg, M. A., \& Casey, E. J. (1989). Object displays do not always support better integrated task performance. Human Factors, 31, 183-198.

Siegel, J. H., Goldwyn, R. M., \& Friedman, H. P. (1971). Pattern and process in the evolution of human septic shock. Surgery, 70, 232-243.

SMITH, L. B., \& KILroy, M. C. (1979). A continuum of dimensional separability. Perception \& Psychophysics, 25, 285-291.

Stokes, A. E., \&ickens, C. D. (1988). Aviation displays. In E. L. Wiener \& D. C. Nagel (Eds.), Human factors in aviation (pp. 387430). New York: Academic Press. 
Treisman, A., \& Paterson, R. (1984). Emergent features, attention, and object perception. Journal of Experimental Psychology: Human Perception \& Performance, 10, 12-31.

TUFTE, E. R. (1983). The visual display of quantitative information. Cheshire, CT: Graphics Press.

Wainer, H., \& Thissen, D. (1981). Graphical data analysis. Annual Review of Psychology, 32, 191-241.

Weintraub, D. J. (1971). Rectangle discriminability: Perceptual relativity and the law of pragnanz. Journal of Experimental Psychology, 88, $1-11$.

WiCkens, C. D. (1986). The object display: Principles and a review of experimental findings (Tech. Rep. CPL-86-6). Champaign: Cognitive Psychophysiology Lab, University of Illinois.
Wickens, C. D., ANDre, A. D. (1988). Proximity compatibility and the object display. Proceedings of the Human Factors Society 32nd Annual Meeting (pp. 1335-1339). Santa Monica, CA: Human Factors Society.

WoOds, D., WiSE, J., \& HANES, L. (1981). An evaluation of nuclear power plant safety parameter display systems. Proceedings of the Human Factors Society 25th Annual Meeting (pp. 110-114). Santa Monica, CA: Human Factors Society.

(Manuscript received December 27, 1988; revision accepted for publication September 1, 1989.) 